\title{
Dynamical behavior of a system of three-dimensional nonlinear difference equations
}

\author{
Inci Okumuş ${ }^{1 *}$ (D) and Yüksel Soykan ${ }^{1}$
}

"Correspondence:

inci_okumus_90@hotmail.com

${ }^{1}$ Department of Mathematics, Art

and Science Faculty, Bülent Ecevit

University, Zonguldak, Turkey

\section{Springer}

\begin{abstract}
In this paper, we study the boundedness, persistence, and periodicity of the positive solutions and the global asymptotic stability of the positive equilibrium points of the system of difference equations

$$
x_{n+1}=A+\frac{x_{n-1}}{z_{n}}, \quad y_{n+1}=A+\frac{y_{n-1}}{z_{n}}, \quad z_{n+1}=A+\frac{z_{n-1}}{y_{n}}, \quad n=0,1, \ldots
$$

where $A \in(0, \infty)$ and the initial conditions $x_{i}, y_{i}, z_{i} \in(0, \infty), i=-1,0$.

MSC: $39 \mathrm{~A} 10 ; 39 \mathrm{~A} 30$

Keywords: System of difference equations; Solution; Boundedness; Equilibrium point; Stability; Global asymptotic stability
\end{abstract}

\section{Introduction}

Difference equation or discrete dynamical system is a diverse field which impacts almost every branch of pure and applied mathematics. Lately, there has been great interest in investigating the behavior of solutions of a system of nonlinear difference equations and discussing the asymptotic stability of their equilibrium points. One of the reasons for this is a necessity for some techniques which can be used in investigating equations arising in mathematical models that describe real life situations in population biology, economics, probability theory, genetics, psychology, and so forth, see [3, 5, 8, 9]. Also, similar works in two and three dimensions (limit behaviors) for more general cases, i.e., continuous and discrete cases, have been done by some authors, see $[1,11-13,16]$. There are many papers in which systems of difference equations have been studied, as in the examples given below.

In [14], Papaschinopoulos and Schinas considered the system of difference equations

$$
x_{n+1}=A+\frac{y_{n}}{x_{n-p}}, \quad y_{n+1}=A+\frac{x_{n}}{y_{n-q}}, \quad n=0,1, \ldots,
$$

where $A \in(0, \infty), p, q$ are positive integers and $x_{-p}, \ldots, x_{0}, y_{-q}, \ldots, y_{0}$ are positive numbers.

(0) The Author(s) 2018. This article is distributed under the terms of the Creative Commons Attribution 4.0 International License (http://creativecommons.org/licenses/by/4.0/), which permits unrestricted use, distribution, and reproduction in any medium, provided you give appropriate credit to the original author(s) and the source, provide a link to the Creative Commons license, and indicate if changes were made. 
In [15], Papaschinopoulos and Schinas studied the system of difference equations

$$
x_{n+1}=A+\frac{x_{n-1}}{y_{n}}, \quad y_{n+1}=A+\frac{y_{n-1}}{x_{n}}, \quad n=0,1, \ldots,
$$

where $A$ is a positive constant and the initial conditions are positive numbers.

In [2], Bao investigated the local stability, oscillation, and boundedness character of positive solutions of the system of difference equations

$$
x_{n+1}=A+\frac{x_{n-1}^{p}}{y_{n}^{p}}, \quad y_{n+1}=A+\frac{y_{n-1}^{p}}{x_{n}^{p}}, \quad n=0,1, \ldots,
$$

where $A \in(0, \infty), p \in[1, \infty)$ and the initial conditions $x_{i}, y_{i} \in(0, \infty), i=-1,0$.

In [7], Gümüș and Soykan considered the dynamical behavior of positive solutions for a system of rational difference equations of the following form:

$$
u_{n+1}=\frac{\alpha u_{n-1}}{\beta+\gamma v_{n-2}^{p}}, \quad v_{n+1}=\frac{\alpha_{1} v_{n-1}}{\beta_{1}+\gamma_{1} u_{n-2}^{p}}, \quad n=0,1, \ldots,
$$

where the parameters $\alpha, \beta, \gamma, \alpha_{1}, \beta_{1}, \gamma_{1}, p$ and the initial values $u_{-i}, v_{-i}$ for $i=0,1,2$ are positive real numbers.

In [6], Göcen and Cebeci studied the general form of periodic solutions of some higher order systems of difference equations

$$
x_{n+1}=\frac{ \pm x_{n-k} y_{n-(2 k+1)}}{y_{n-(2 k+1)} \mp y_{n-k}}, \quad y_{n+1}=\frac{ \pm y_{n-k} x_{n-(2 k+1)}}{x_{n-(2 k+1)} \mp x_{n-k}}, \quad n, k \in \mathbb{N}_{0} \text {, }
$$

where the initial values are arbitrary real numbers.

Also, for similar results in the area of difference equations and systems, see $[4,10,16-$ 21].

In this paper, we investigate the stability, boundedness character, and periodicity of positive solutions of the system of difference equations

$$
\begin{aligned}
& x_{n+1}=A+\frac{x_{n-1}}{z_{n}}, \quad y_{n+1}=A+\frac{y_{n-1}}{z_{n}}, \\
& z_{n+1}=A+\frac{z_{n-1}}{y_{n}}, \quad n=0,1, \ldots,
\end{aligned}
$$

where $A$ and the initial values $x_{-1}, x_{0}, y_{-1}, y_{0}, z_{-1}, z_{0}$ are positive real numbers.

\section{Preliminaries}

We recall some basic definitions that we afterwards need in the paper.

Let us introduce the discrete dynamical system:

$$
\begin{aligned}
& x_{n+1}=f_{1}\left(x_{n}, x_{n-1}, \ldots, x_{n-k}, y_{n}, y_{n-1}, \ldots, y_{n-k}, z_{n}, z_{n-1}, \ldots, z_{n-k}\right), \\
& y_{n+1}=f_{2}\left(x_{n}, x_{n-1}, \ldots, x_{n-k}, y_{n}, y_{n-1}, \ldots, y_{n-k}, z_{n}, z_{n-1}, \ldots, z_{n-k}\right), \\
& z_{n+1}=f_{3}\left(x_{n}, x_{n-1}, \ldots, x_{n-k}, y_{n}, y_{n-1}, \ldots, y_{n-k}, z_{n}, z_{n-1}, \ldots, z_{n-k}\right),
\end{aligned}
$$

$n \in \mathbb{N}$, where $f_{1}: I_{1}^{k+1} \times I_{2}^{k+1} \times I_{3}^{k+1} \rightarrow I_{1}, f_{2}: I_{1}^{k+1} \times I_{2}^{k+1} \times I_{3}^{k+1} \rightarrow I_{2}$, and $f_{3}: I_{1}^{k+1} \times I_{2}^{k+1} \times$ $I_{3}^{k+1} \rightarrow I_{3}$ are continuously differentiable functions and $I_{1}, I_{2}, I_{3}$ are some intervals of real 
numbers. Also, a solution $\left\{\left(x_{n}, y_{n}, z_{n}\right)\right\}_{n=-k}^{\infty}$ of system (7) is uniquely determined by the initial values $\left(x_{-i}, y_{-i}, z_{-i}\right) \in I_{1} \times I_{2} \times I_{3}$ for $i \in\{0,1, \ldots, k\}$.

Definition 1 An equilibrium point of system (7) is a point $(\bar{x}, \bar{y}, \bar{z})$ that satisfies

$$
\begin{aligned}
& \bar{x}=f_{1}(\bar{x}, \bar{x}, \ldots, \bar{x}, \bar{y}, \bar{y}, \ldots, \bar{y}, \bar{z}, \bar{z}, \ldots, \bar{z}), \\
& \bar{y}=f_{2}(\bar{x}, \bar{x}, \ldots, \bar{x}, \bar{y}, \bar{y}, \ldots, \bar{y}, \bar{z}, \bar{z}, \ldots, \bar{z}), \\
& \bar{z}=f_{3}(\bar{x}, \bar{x}, \ldots, \bar{x}, \bar{y}, \bar{y}, \ldots, \bar{y}, \bar{z}, \bar{z}, \ldots, \bar{z}) .
\end{aligned}
$$

Together with system (7), if we consider the associated vector map

$$
F=\left(f_{1}, x_{n}, x_{n-1}, \ldots, x_{n-k}, f_{2}, y_{n}, y_{n-1}, \ldots, y_{n-k}, f_{3}, z_{n-1}, \ldots, z_{n-k}\right)
$$

then the point $(\bar{x}, \bar{y}, \bar{z})$ is also called a fixed point of the vector map $F$.

Definition $2([2,3])$ Let $(\bar{x}, \bar{y}, \bar{z})$ be an equilibrium point of system (7).

(a) An equilibrium point $(\bar{x}, \bar{y}, \bar{z})$ is called stable if, for every $\varepsilon>0$, there exists $\delta>0$ such that, for every initial value $\left(x_{-i}, y_{-i}, z_{-i}\right) \in I_{1} \times I_{2} \times I_{3}$, with

$$
\sum_{i=-k}^{0}\left|x_{i}-\bar{x}\right|<\delta, \quad \sum_{i=-k}^{0}\left|y_{i}-\bar{y}\right|<\delta, \quad \sum_{i=-k}^{0}\left|z_{i}-\bar{z}\right|<\delta
$$

implying $\left|x_{n}-\bar{x}\right|<\varepsilon,\left|y_{n}-\bar{y}\right|<\varepsilon,\left|z_{n}-\bar{z}\right|<\varepsilon$ for $n \in \mathbb{N}$.

(b) An equilibrium point $(\bar{x}, \bar{y}, \bar{z})$ of system (7) is called unstable if it is not stable.

(c) An equilibrium point $(\bar{x}, \bar{y}, \bar{z})$ of system (7) is called locally asymptotically stable if it is stable and if, in addition, there exists $\gamma>0$ such that

$$
\sum_{i=-k}^{0}\left|x_{i}-\bar{x}\right|<\gamma, \quad \sum_{i=-k}^{0}\left|y_{i}-\bar{y}\right|<\gamma, \quad \sum_{i=-k}^{0}\left|z_{i}-\bar{z}\right|<\gamma
$$

and $\left(x_{n}, y_{n}, z_{n}\right) \rightarrow(\bar{x}, \bar{y}, \bar{z})$ as $n \rightarrow \infty$.

(d) An equilibrium point $(\bar{x}, \bar{y}, \bar{z})$ of system (7) is called a global attractor if $\left(x_{n}, y_{n}, z_{n}\right) \rightarrow(\bar{x}, \bar{y}, \bar{z})$ as $n \rightarrow \infty$.

(e) An equilibrium point $(\bar{x}, \bar{y}, \bar{z})$ of system (7) is called globally asymptotically stable if it is stable and a global attractor.

Definition 3 Let $(\bar{x}, \bar{y}, \bar{z})$ be an equilibrium point of the map $F$ where $f_{1}, f_{2}$, and $f_{3}$ are continuously differentiable functions at $(\bar{x}, \bar{y}, \bar{z})$. The linearized system of system (7) about the equilibrium point $(\bar{x}, \bar{y}, \bar{z})$ is

$$
X_{n+1}=F\left(X_{n}\right)=B X_{n}
$$


where

$$
X_{n}=\left(\begin{array}{c}
x_{n} \\
\vdots \\
x_{n-k} \\
y_{n} \\
\vdots \\
y_{n-k} \\
z_{n} \\
\vdots \\
z_{n-k}
\end{array}\right)
$$

and $B$ is a Jacobian matrix of system (7) about the equilibrium point $(\bar{x}, \bar{y}, \bar{z})$.

Definition 4 Assume that $X_{n+1}=F\left(X_{n}\right), n=0,1, \ldots$, is a system of difference equations such that $\bar{X}$ is a fixed point of $F$. If no eigenvalues of the Jacobian matrix $B$ about $\bar{X}$ have absolute value equal to one, then $\bar{X}$ is called hyperbolic. Otherwise, $\bar{X}$ is said to be nonhyperbolic.

Theorem 5 (The linearized stability theorem [8], p. 11) Assume that

$$
X_{n+1}=F\left(X_{n}\right), \quad n=0,1, \ldots
$$

is a system of difference equations such that $\bar{X}$ is a fixed point of $F$.

(a) If all eigenvalues of the Jacobian matrix $B$ about $\bar{X}$ lie inside the open unit disk

$|\lambda|<1$, that is, if all of them have absolute value less than one, then $\bar{X}$ is locally asymptotically stable.

(b) If at least one of them has a modulus greater than one, then $\bar{X}$ is unstable.

A positive solution $\left\{\left(x_{n}, y_{n}, z_{n}\right)\right\}_{n=-k}^{\infty}$ of system (7) is bounded and persists if there exist positive constants $M, N$ such that

$$
M \leq x_{n}, y_{n}, z_{n} \leq N, \quad n=-m,-m+1, \ldots
$$

A positive solution $\left\{\left(x_{n}, y_{n}, z_{n}\right)\right\}_{n=-k}^{\infty}$ of system (7) is periodic with period $p$ if

$$
x_{n+p}=x_{n}, \quad y_{n+p}=y_{n}, \quad z_{n+p}=z_{n} \quad \text { for all } n \geq-1 .
$$

\section{Main results}

In this section, we prove our main results.

Theorem 6 The following statements are true:

(i) If $(\bar{x}, \bar{y}, \bar{z})$ is a positive equilibrium point of system (6), then

$$
(\bar{x}, \bar{y}, \bar{z})= \begin{cases}(A+1, A+1, A+1), & \text { if } A \neq 1, \\ \left(\mu, \mu, \frac{\mu}{\mu-1}\right), \quad \mu \in(1, \infty) & \text { if } A=1\end{cases}
$$


(ii) If $A>1$, then the equilibrium point of system (6) is locally asymptotically stable.

(iii) If $0<A<1$, then the equilibrium point of system (6) is locally unstable.

(iv) If $A=1$, then for every $\mu \in(1, \infty)$ there exist positive solutions $\left\{\left(x_{n}, y_{n}, z_{n}\right)\right\}$ of system (6) which tend to the positive equilibrium point $\left(\mu, \mu, \frac{\mu}{\mu-1}\right)$.

Proof (i) It is easily seen from the definition of equilibrium point that the equilibrium points of system (6) are the nonnegative solutions of the equations

$$
\bar{x}=A+\frac{\bar{x}}{\bar{z}}, \quad \bar{y}=A+\frac{\bar{y}}{\bar{z}}, \quad \bar{z}=A+\frac{\bar{z}}{\bar{y}} .
$$

From this, we get

$$
\begin{gathered}
\bar{x} \cdot \bar{z}=A \bar{z}+\bar{x}, \quad \bar{y} \cdot \bar{z}=A \bar{z}+\bar{y}, \quad \bar{z} \cdot \bar{y}=A \bar{y}+\bar{z} \\
\Rightarrow \quad \bar{x} \cdot \bar{z}-\bar{x}=\bar{y} \cdot \bar{z}-\bar{y}, \quad A \bar{z}+\bar{y}=A \bar{y}+\bar{z} \\
\Rightarrow \quad \bar{x}(\bar{z}-1)=\bar{y}(\bar{z}-1), \quad \bar{z}(A-1)=\bar{y}(A-1) .
\end{gathered}
$$

From which it follows that if $A \neq 1$,

$$
\bar{x}=\bar{y}=\bar{z}=A+1 \quad \Rightarrow \quad(\bar{x}, \bar{y}, \bar{z})=(A+1, A+1, A+1) .
$$

Also, we have

$$
\begin{array}{cc}
\frac{\bar{x} \cdot \bar{z}-\bar{x}}{\bar{z}}=A, \quad \frac{\bar{y} \cdot \bar{z}-\bar{y}}{\bar{z}}=A, & \frac{\bar{z} \cdot \bar{y}-\bar{z}}{\bar{y}}=A \\
\Rightarrow \quad \frac{\bar{x} \cdot \bar{z}-\bar{x}}{\bar{z}}=\frac{\bar{y} \cdot \bar{z}-\bar{y}}{\bar{z}}, & \frac{\bar{y} \cdot \bar{z}-\bar{y}}{\bar{z}}=\frac{\bar{z} \cdot \bar{y}-\bar{z}}{\bar{y}} \\
\Rightarrow \quad \bar{x} \cdot \bar{z}-\bar{x}=\bar{y} \cdot \bar{z}-\bar{y}, & \bar{y}^{2} \bar{z}-\bar{y}^{2}=\bar{z}^{2} \bar{y}-\bar{z}^{2} \\
\Rightarrow \quad \bar{x}(\bar{z}-1)=\bar{y}(\bar{z}-1), & \bar{y} \cdot \bar{z}(\bar{y}-\bar{z})=(\bar{y}-\bar{z})(\bar{y}+\bar{z}) .
\end{array}
$$

From which it follows that if $A=1$,

$$
\begin{aligned}
\bar{x} & =\bar{y} \quad \text { and } \bar{y} \cdot \bar{z}=\bar{y}+\bar{z} \\
& \Rightarrow \quad(\bar{x}, \bar{y}, \bar{z})=\left(\mu, \mu, \frac{\mu}{\mu-1}\right), \quad \mu \in(1, \infty) .
\end{aligned}
$$

In that case, we have a continuum of positive equilibria which lie on the hyperboloid

$$
\bar{y} \cdot \bar{z}=\bar{y}+\bar{z}
$$

(ii) We consider the following transformation to build the corresponding linearized form of system (6):

$$
\left(x_{n}, x_{n-1}, y_{n}, y_{n-1}, z_{n}, z_{n-1}\right) \rightarrow\left(f, f_{1}, g, g_{1}, h, h_{1}\right),
$$


where

$$
\begin{aligned}
& f=A+\frac{x_{n-1}}{z_{n}}, \\
& f_{1}=x_{n}, \\
& g=A+\frac{y_{n-1}}{z_{n}}, \\
& g_{1}=y_{n}, \\
& h=A+\frac{z_{n-1}}{y_{n}}, \\
& h_{1}=z_{n} .
\end{aligned}
$$

The Jacobian matrix about the equilibrium point $(\bar{x}, \bar{y}, \bar{z})$ under the above transformation is given by

$$
B(\bar{x}, \bar{y}, \bar{z})=\left(\begin{array}{cccccc}
0 & \frac{1}{\bar{z}} & 0 & 0 & -\frac{\bar{x}}{\bar{z}^{2}} & 0 \\
1 & 0 & 0 & 0 & 0 & 0 \\
0 & 0 & 0 & \frac{1}{\bar{z}} & -\frac{\bar{y}}{\bar{z}^{2}} & 0 \\
0 & 0 & 1 & 0 & 0 & 0 \\
0 & 0 & -\frac{\bar{z}}{\bar{y}^{2}} & 0 & 0 & \frac{1}{\bar{y}} \\
0 & 0 & 0 & 0 & 1 & 0
\end{array}\right) .
$$

Hence, the linearized system of system (6) about the equilibrium point $(\bar{x}, \bar{y}, \bar{z})=(A+1$, $A+1, A+1)$ is

$$
X_{n+1}=B(\bar{x}, \bar{y}, \bar{z}) X_{n},
$$

where

$$
X_{n}=\left(\left(x_{n}, x_{n-1}, y_{n}, y_{n-1}, z_{n}, z_{n-1}\right)\right)^{T}
$$

and

$$
B(\bar{x}, \bar{y}, \bar{z})=\left(\begin{array}{cccccc}
0 & \frac{1}{A+1} & 0 & 0 & -\frac{1}{A+1} & 0 \\
1 & 0 & 0 & 0 & 0 & 0 \\
0 & 0 & 0 & \frac{1}{A+1} & -\frac{1}{A+1} & 0 \\
0 & 0 & 1 & 0 & 0 & 0 \\
0 & 0 & -\frac{1}{A+1} & 0 & 0 & \frac{1}{A+1} \\
0 & 0 & 0 & 0 & 1 & 0
\end{array}\right) .
$$

Then the characteristic equation of $B(\bar{x}, \bar{y}, \bar{z})$ about $(\bar{x}, \bar{y}, \bar{z})=(A+1, A+1, A+1)$ is

$$
\lambda^{6}-\frac{(3 A+4)}{(A+1)^{2}} \lambda^{4}+\frac{(3 A+4)}{(A+1)^{3}} \lambda^{2}-\frac{1}{(A+1)^{3}}=0 .
$$

From this, the roots of characteristic equation (10) are

$$
\lambda_{1}=\frac{1}{\sqrt{A+1}},
$$




$$
\begin{aligned}
& \lambda_{2}=-\frac{1}{\sqrt{A+1}}, \\
& \lambda_{3}=\frac{1}{2} \frac{\sqrt{4 A+5}-1}{A+1}, \\
& \lambda_{4}=-\frac{1}{2} \frac{\sqrt{4 A+5}+1}{A+1}, \\
& \lambda_{5}=\frac{1}{2} \frac{\sqrt{4 A+5}+1}{A+1}, \\
& \lambda_{6}=-\frac{1}{2} \frac{\sqrt{4 A+5}-1}{A+1} .
\end{aligned}
$$

From the linearized stability theorem, since $A>1$, all roots of the characteristic equation lie inside the open unit disk $|\lambda|<1$. Therefore, the positive equilibrium point of system (6) is locally asymptotically stable.

(iii) From the proof of (ii), it is true.

(iv) From (9), the linearized system of system (6) about the equilibrium point $(\bar{x}, \bar{y}, \bar{z})=$ $\left(\mu, \mu, \frac{\mu}{\mu-1}\right)$ is

$$
X_{n+1}=B(\bar{x}, \bar{y}, \bar{z}) X_{n},
$$

where

$$
X_{n}=\left(\left(x_{n}, x_{n-1}, y_{n}, y_{n-1}, z_{n}, z_{n-1}\right)\right)^{T}
$$

and

$$
B(\bar{x}, \bar{y}, \bar{z})=\left(\begin{array}{cccccc}
0 & \frac{\mu-1}{\mu} & 0 & 0 & -\frac{(\mu-1)^{2}}{\mu} & 0 \\
1 & 0 & 0 & 0 & 0 & 0 \\
0 & 0 & 0 & \frac{\mu-1}{\mu} & -\frac{(\mu-1)^{2}}{\mu} & 0 \\
0 & 0 & 1 & 0 & 0 & 0 \\
0 & 0 & -\frac{1}{\mu(\mu-1)} & 0 & 0 & \frac{1}{\mu} \\
0 & 0 & 0 & 0 & 1 & 0
\end{array}\right) .
$$

Hence, the characteristic equation of the matrix $B$ is

$$
\lambda^{6}-\left(\frac{2 \mu^{2}-1}{\mu^{2}}\right) \lambda^{4}+\left(\frac{\mu^{3}+\mu^{2}-3 \mu+1}{\mu}\right) \lambda^{2}-\frac{(\mu-1)^{2}}{\mu^{3}}=0 .
$$

Therefore, the roots of equation (11) are:

$$
\begin{aligned}
& \lambda_{1}=-1, \\
& \lambda_{2}=1, \\
& \lambda_{3}=\frac{\sqrt{\mu-1}}{\mu}, \\
& \lambda_{4}=-\frac{\sqrt{\mu-1}}{\mu},
\end{aligned}
$$




$$
\begin{aligned}
& \lambda_{5}=\sqrt{\frac{\mu-1}{\mu}}, \\
& \lambda_{6}=-\sqrt{\frac{\mu-1}{\mu}} .
\end{aligned}
$$

Then the modulus of four of the roots of (11) is less than 1 . So, there exist positive solutions of system (6) which tend to the positive equilibrium point $\left(\mu, \mu, \frac{\mu}{\mu-1}\right)$ of system (6) (this follows from the following proposition). This completes the proof.

In the following proposition we find positive solutions of system (6) which tend to $(\bar{x}, \bar{y}, \bar{z})$ as $n \rightarrow \infty$.

Proposition 7 Let $\left\{\left(x_{n}, y_{n}, z_{n}\right)\right\}$ be a positive solution of system (6). Then, if there exists $s \in\{-1,0, \ldots\}$ such that, for $n \geq s, x_{n} \geq \bar{x}, y_{n} \geq \bar{y}, z_{n} \geq \bar{z}$ (resp., $x_{n}<\bar{x}, y_{n}<\bar{y}, z_{n}<\bar{z}$ ), the solution $\left\{\left(x_{n}, y_{n}, z_{n}\right)\right\}$ tends to the positive equilibrium $(\bar{x}, \bar{y}, \bar{z})$ of system (6) as $n \rightarrow \infty$.

Proof Let $\left\{\left(x_{n}, y_{n}, z_{n}\right)\right\}$ be a positive solution of system (6) such that

$$
x_{n} \geq \bar{x}, \quad y_{n} \geq \bar{y}, \quad z_{n} \geq \bar{z}, \quad n \geq s,
$$

where $s \in\{-1,0, \ldots\}$. Then from (6) and (12) we have

$$
x_{n+1}=A+\frac{x_{n-1}}{z_{n}} \leq A+\frac{x_{n-1}}{\bar{z}}, \quad n \geq 1 .
$$

Set

$$
u_{n+1}=A+\frac{u_{n-1}}{\bar{z}}, \quad n \geq 1
$$

such that

$$
u_{s}=x_{s}, \quad u_{s+1}=x_{s+1}, \quad s \in\{-1,0,1, \ldots\}, n \geq s .
$$

Then the solution $u_{n}$ of the difference equation (14) is as follows:

$$
u_{n}=c_{1}\left(\frac{1}{\sqrt{\bar{z}}}\right)^{n}+c_{1}\left(-\frac{1}{\sqrt{\bar{z}}}\right)^{n}+\frac{A \bar{z}}{A \bar{z}-1}=c_{1}\left(\frac{1}{\sqrt{\bar{z}}}\right)^{n}+c_{1}\left(-\frac{1}{\sqrt{\bar{z}}}\right)^{n}+\bar{x}
$$

where $c_{1}, c_{2}$ depend on $x_{s}, x_{s+1}$. In addition, relations (13) and (14) imply that

$$
x_{n+1}-u_{n+1} \leq \frac{x_{n-1}-u_{n-1}}{\bar{z}}, \quad n>s .
$$

Then, by using (15) and (17) and induction, we have

$$
x_{n} \leq u_{n}, \quad n \geq s .
$$

Therefore, from (12), (16), and (18), it is clear that

$$
\lim _{n \rightarrow \infty} x_{n}=\bar{x}
$$


Similarly, we can prove that

$$
\lim _{n \rightarrow \infty} y_{n}=\bar{y} \quad \text { and } \quad \lim _{n \rightarrow \infty} z_{n}=\bar{z} .
$$

Thus, from (19) and (20), the solution $\left\{\left(x_{n}, y_{n}, z_{n}\right)\right\}$ tends to $(\bar{x}, \bar{y}, \bar{z})$ as $n \rightarrow \infty$.

Arguing as above, we can show that if $x_{n}<\bar{x}, y_{n}<\bar{y}, z_{n}<\bar{z}$ for $n \geq s$, then $\left\{\left(x_{n}, y_{n}, z_{n}\right)\right\}$ tends to $(\bar{x}, \bar{y}, \bar{z})$ as $n \rightarrow \infty$. The proof of the proposition is completed.

Theorem 8 Assume that $0<A<1$ and $\left\{\left(x_{n}, y_{n}, z_{n}\right)\right\}$ is an arbitrary positive solution of system (6). Then the following statements are true.

(i) If

$$
\begin{aligned}
& x_{-1}<1, \quad y_{-1}<1, \quad z_{-1}<1, \quad x_{0}>\frac{1}{1-A}, \\
& y_{0}>\frac{1}{1-A}, \quad z_{0}>\frac{1}{1-A},
\end{aligned}
$$

then

$$
\begin{aligned}
& \lim _{n \rightarrow \infty} x_{2 n+1}=A, \quad \lim _{n \rightarrow \infty} y_{2 n+1}=A, \quad \lim _{n \rightarrow \infty} z_{2 n+1}=A \text {, } \\
& \lim _{n \rightarrow \infty} x_{2 n}=\infty, \quad \lim _{n \rightarrow \infty} y_{2 n}=\infty, \quad \lim _{n \rightarrow \infty} z_{2 n}=\infty .
\end{aligned}
$$

(ii) If

$$
\begin{aligned}
& x_{0}<1, \quad y_{0}<1, \quad z_{0}<1, \quad x_{-1}>\frac{1}{1-A}, \\
& y_{-1}>\frac{1}{1-A}, \quad z_{-1}>\frac{1}{1-A},
\end{aligned}
$$

then

$$
\begin{aligned}
& \lim _{n \rightarrow \infty} x_{2 n+1}=\infty, \quad \lim _{n \rightarrow \infty} y_{2 n+1}=\infty, \quad \lim _{n \rightarrow \infty} z_{2 n+1}=\infty, \\
& \lim _{n \rightarrow \infty} x_{2 n}=A, \quad \lim _{n \rightarrow \infty} y_{2 n}=A, \quad \lim _{n \rightarrow \infty} z_{2 n}=A .
\end{aligned}
$$

Proof (i) From (6) and (21), we get

$$
\begin{aligned}
& x_{1}=A+\frac{x_{-1}}{z_{0}}<A+\frac{1}{z_{0}}<A+(1-A)=1, \\
& y_{1}=A+\frac{y_{-1}}{z_{0}}<A+\frac{1}{z_{0}}<A+(1-A)=1, \\
& z_{1}=A+\frac{z_{-1}}{y_{0}}<A+\frac{1}{y_{0}}<A+(1-A)=1, \\
& x_{2}=A+\frac{x_{0}}{z_{1}}>x_{0}>\frac{1}{1-A}, \\
& y_{2}=A+\frac{y_{0}}{z_{1}}>y_{0}>\frac{1}{1-A}, \\
& z_{2}=A+\frac{z_{0}}{y_{1}}>z_{0}>\frac{1}{1-A} .
\end{aligned}
$$


By induction, for $n=0,1,2, \ldots$, we obtain

$$
\begin{aligned}
& x_{2 n-1}<1, \quad y_{2 n-1}<1, \quad z_{2 n-1}<1, \\
& x_{2 n}>\frac{1}{1-A}, \quad y_{2 n}>\frac{1}{1-A}, \quad z_{2 n}>\frac{1}{1-A} .
\end{aligned}
$$

Thus, relations (6) and (23) imply that

$$
\begin{aligned}
& x_{2 n}=A+\frac{x_{2 n-2}}{z_{2 n-1}}>A+x_{2 n-2}>2 A+\frac{x_{2 n-4}}{z_{2 n-3}}>2 A+x_{2 n-4}, \\
& y_{2 n}=A+\frac{y_{2 n-2}}{z_{2 n-1}}>A+y_{2 n-2}>2 A+\frac{y_{2 n-4}}{z_{2 n-3}}>2 A+y_{2 n-4}, \\
& z_{2 n}=A+\frac{z_{2 n-2}}{y_{2 n-1}}>A+z_{2 n-2}>2 A+\frac{z_{2 n-4}}{y_{2 n-3}}>2 A+z_{2 n-4} .
\end{aligned}
$$

From which we get

$$
\lim _{n \rightarrow \infty} x_{2 n}=\infty, \quad \lim _{n \rightarrow \infty} y_{2 n}=\infty, \quad \lim _{n \rightarrow \infty} z_{2 n}=\infty
$$

Noting that (23) and taking limits on both sides of three equations

$$
x_{2 n+1}=A+\frac{x_{2 n-1}}{z_{2 n}}, \quad y_{2 n+1}=A+\frac{y_{2 n-1}}{z_{2 n}}, \quad z_{2 n+1}=A+\frac{z_{2 n-1}}{y_{2 n}},
$$

we have

$$
\lim _{n \rightarrow \infty} x_{2 n+1}=A, \quad \lim _{n \rightarrow \infty} y_{2 n+1}=A, \quad \lim _{n \rightarrow \infty} z_{2 n+1}=A
$$

(ii) The proof is similar to the proof of (i), so we leave it to readers.

Theorem 9 Assume that $A=1$. Then every positive solution of system (6) $i$ sounded and persists.

Proof Let $\left\{\left(x_{n}, y_{n}, z_{n}\right)\right\}$ be a positive solution of system (6).

Obviously, $x_{n}>1, y_{n}>1, z_{n}>1$, for $n \geq 1$. So, we have

$$
x_{i}, y_{i}, z_{i} \in\left[K, \frac{K}{K-1}\right], \quad i=1,2, \ldots, m+1,
$$

where

$$
K=\min \left\{\alpha, \frac{\beta}{\beta-1}\right\}>1, \quad \alpha=\min _{1 \leq i \leq m+1}\left\{x_{i}, y_{i}, z_{i}\right\}, \beta=\max _{1 \leq i \leq m+1}\left\{x_{i}, y_{i}, z_{i}\right\} .
$$

Then we obtain

$$
\begin{aligned}
& K=1+\frac{K}{K /(K-1)} \leq x_{m+2}=1+\frac{x_{1}}{z_{m+1}} \leq 1+\frac{K /(K-1)}{K}=\frac{K}{K-1}, \\
& K=1+\frac{K}{K /(K-1)} \leq y_{m+2}=1+\frac{y_{1}}{z_{m+1}} \leq 1+\frac{K /(K-1)}{K}=\frac{K}{K-1}, \\
& K=1+\frac{K}{K /(K-1)} \leq z_{m+2}=1+\frac{z_{1}}{y_{m+1}} \leq 1+\frac{K /(K-1)}{K}=\frac{K}{K-1} .
\end{aligned}
$$


By induction, we get

$$
x_{i}, y_{i}, z_{i} \in\left[K, \frac{K}{K-1}\right], \quad i=1,2, \ldots
$$

The proof of the following theorem is seen easily and will be omitted.

Theorem 10 Assume $A=1$. Then every positive solution of system (6) is periodic of pe$\operatorname{riod} 2$.

Theorem 11 Assume $A>1$. Then every positive solution of system (6) is bounded.

Proof Let $\left\{\left(x_{n}, y_{n}, z_{n}\right)\right\}$ be a positive solution of system (6). Clearly,

$$
x_{n}, y_{n}, z_{n}>A>1 \text { for } n \geq 1 \text {. }
$$

From (24), we have

$$
x_{n+1}=A+\frac{x_{n-1}}{z_{n}} \leq A+\frac{x_{n-1}}{A}, \quad n \geq 1
$$

Set

$$
u_{n+1}=A+\frac{u_{n-1}}{A}, \quad n \geq 1,
$$

such that

$$
u_{s}=x_{s}, \quad u_{s+1}=x_{s+1}, \quad s \in\{-1,0,1, \ldots\}, n \geq s .
$$

Then the solution $u_{n}$ of the difference equation (26) is as follows:

$$
u_{n}=c_{1}\left(\frac{1}{\sqrt{A}}\right)^{n}+c_{1}\left(-\frac{1}{\sqrt{A}}\right)^{n}+\frac{A^{2}}{A-1} \text {. }
$$

Indeed, from (26) we get

$$
\begin{aligned}
& u_{n+1}-\frac{1}{A} u_{n-1}=0 \Rightarrow \lambda^{2}-\frac{1}{A}=0 \\
& \Rightarrow \lambda_{1,2}= \pm \frac{1}{\sqrt{A}} .
\end{aligned}
$$

The homogeneous solution of difference equation (26) is given by

$$
u_{h}=c_{1}\left(\frac{1}{\sqrt{A}}\right)^{n}+c_{2}\left(-\frac{1}{\sqrt{A}}\right)^{n} \text {. }
$$

Also, from (26), the equilibrium solution of difference equation (26) is as follows:

$$
\bar{x}-\frac{1}{A} \bar{x}=A \quad \Rightarrow \quad \bar{x}=\frac{A^{2}}{A-1} .
$$


In addition, relations (25) and (28) imply that

$$
x_{n+1}-u_{n+1} \leq \frac{x_{n-1}-u_{n-1}}{A}, \quad n>s .
$$

Then, by using (27) and (29) and induction, we have

$$
x_{n} \leq u_{n}, \quad n \geq s
$$

Therefore, from (24), (28), and (30), we obtain

$$
A<x_{n} \leq c_{1}\left(\frac{1}{\sqrt{A}}\right)^{n}+c_{2}\left(-\frac{1}{\sqrt{A}}\right)^{n}+\frac{A^{2}}{A-1}
$$

where

$$
\begin{aligned}
& c_{1}=\frac{1}{2}\left(x_{0}+\sqrt{A} x_{1}-\frac{A^{2}}{A-1}(1+\sqrt{A})\right), \\
& c_{2}=\frac{1}{2}\left(x_{0}-\sqrt{A} x_{1}-\frac{A^{2}}{A-1}(1-\sqrt{A})\right) .
\end{aligned}
$$

Similarly, we can prove that

$$
\begin{aligned}
& A<y_{n} \leq c_{3}\left(\frac{1}{\sqrt{A}}\right)^{n}+c_{4}\left(-\frac{1}{\sqrt{A}}\right)^{n}+\frac{A^{2}}{A-1}, \\
& A<z_{n} \leq c_{5}\left(\frac{1}{\sqrt{A}}\right)^{n}+c_{6}\left(-\frac{1}{\sqrt{A}}\right)^{n}+\frac{A^{2}}{A-1},
\end{aligned}
$$

where

$$
\begin{aligned}
& c_{3}=\frac{1}{2}\left(y_{0}+\sqrt{A} y_{1}-\frac{A^{2}}{A-1}(1+\sqrt{A})\right), \\
& c_{4}=\frac{1}{2}\left(y_{0}-\sqrt{A} y_{1}-\frac{A^{2}}{A-1}(1-\sqrt{A})\right), \\
& c_{5}=\frac{1}{2}\left(z_{0}+\sqrt{A} z_{1}-\frac{A^{2}}{A-1}(1+\sqrt{A})\right), \\
& c_{6}=\frac{1}{2}\left(z_{0}-\sqrt{A} z_{1}-\frac{A^{2}}{A-1}(1-\sqrt{A})\right) .
\end{aligned}
$$

Theorem 12 Suppose that $A>1$. Then the positive equilibrium point of system (6) is globally asymptotically stable.

Proof By means of Theorem 11, we set

$$
\begin{array}{lll}
L_{1}=\lim _{n \rightarrow \infty} \sup x_{n}, & L_{2}=\lim _{n \rightarrow \infty} \sup y_{n}, & L_{3}=\lim _{n \rightarrow \infty} \sup z_{n}, \\
m_{1}=\lim _{n \rightarrow \infty} \inf x_{n}, & m_{2}=\lim _{n \rightarrow \infty} \inf y_{n}, & m_{3}=\lim _{n \rightarrow \infty} \inf z_{n} .
\end{array}
$$


Then, from (6) and (31), we have

$$
\begin{aligned}
& L_{1} \leq A+\frac{L_{1}}{m_{3}}, \quad L_{2} \leq A+\frac{L_{2}}{m_{3}}, \quad L_{3} \leq A+\frac{L_{3}}{m_{2}}, \\
& m_{1} \geq A+\frac{m_{1}}{L_{3}}, \quad m_{2} \geq A+\frac{m_{2}}{L_{3}}, \quad m_{3} \geq A+\frac{m_{3}}{L_{2}} .
\end{aligned}
$$

Relations (32) imply that

$$
A L_{2}+m_{3} \leq m_{3} L_{2} \leq A m_{3}+L_{2}, \quad A L_{3}+m_{2} \leq m_{2} L_{3} \leq A m_{2}+L_{3}
$$

from which we have

$$
(A-1)\left(L_{2}-m_{3}\right) \leq 0, \quad(A-1)\left(L_{3}-m_{2}\right) \leq 0 .
$$

Since $A>1$, we get

$$
L_{2} \leq m_{3} \leq L_{3}, \quad L_{3} \leq m_{2} \leq L_{2}
$$

from this it is obvious that

$$
L_{2}=L_{3}=m_{2}=m_{3} \text {. }
$$

Moreover, from (32) it follows that

$$
L_{1} m_{3} \leq A m_{3}+L_{1}, \quad m_{1} L_{3} \leq A L_{3}+m_{1},
$$

from which

$$
L_{1}\left(m_{3}-1\right) \leq A m_{3}, \quad A L_{3} \leq m_{1}\left(L_{3}-1\right) .
$$

Using (33), we have

$$
L_{1}\left(L_{3}-1\right) \leq m_{1}\left(L_{3}-1\right),
$$

then

$$
L_{1} \leq m_{1} \text {. }
$$

Since $x_{n}$ is bounded, it implies that

$$
L_{1}=m_{1} \text {. }
$$

Hence, every positive solution $\left\{\left(x_{n}, y_{n}, z_{n}\right)\right\}$ of system (6) tends to the positive equilibrium system (6). So, the proof is completed. 


\section{Future works}

We will concentrate on the dynamical behavior of the following system of difference equations:

$$
x_{n+1}=A+\frac{x_{n-m}}{z_{n}}, \quad y_{n+1}=A+\frac{y_{n-m}}{z_{n}}, \quad z_{n+1}=A+\frac{z_{n-m}}{y_{n}}, \quad n=0,1, \ldots
$$

where $A \in(0, \infty)$ and $x_{i}, y_{i}, z_{i} \in(0, \infty), i=0,1, \ldots, m$, and the following cyclic system of difference equations:

$$
x_{n+1}^{(i)}=A_{i}+\frac{x_{n-1}^{(i)}}{x_{n}^{(i+1)}}, \quad i=1,2, \ldots, k
$$

\section{Acknowledgements}

We are thankful to the referees for their careful reading of the manuscript and for valuable comments and suggestions that greatly improved the presentation of this work.

\section{Funding}

Not applicable.

\section{Competing interests}

The authors declare that they have no competing interests.

\section{Authors' contributions}

All authors contributed equally to the writing of this paper. All authors read and approved the final manuscript.

\section{Publisher's Note}

Springer Nature remains neutral with regard to jurisdictional claims in published maps and institutional affiliations.

Received: 23 February 2018 Accepted: 29 May 2018 Published online: 26 June 2018

\section{References}

1. Akın, E., Öztürk, Ö.: Limiting behaviors of nonoscillatory solutions for two-dimensional nonlinear time scale systems. Mediterr. J. Math. 14, 34 (2017)

2. Bao, H.: Dynamical behavior of a system of second-order nonlinear difference equations. Int. J. Differ. Equ. 2015 Article ID 679017 (2015)

3. Camouzis, E., Ladas, G.: Dynamics of Third-Order Rational Difference Equations with Open Problems and Conjecture. Chapman \& Hall, London (2008)

4. Camouzis, E., Papaschinopoulos, G.: Global asymptotic behavior of positive solutions on the system of rational difference equations $x_{n+1}=1+\frac{x_{n}}{y_{n-m}}, y_{n+1}=1+\frac{y_{n}}{x_{n-m}}$. Appl. Math. Lett. 17(6), 733-737 (2004)

5. Elaydi, S.: An Introduction to Difference Equations. Springer, New York (2015)

6. Göcen, M., Cebeci, A.: On the periodic solutions of some systems of higher order difference equations. Rocky Mt. J. Math. (2018) To appear

7. Gümüş, M., Soykan, Y.: Global character of a six-dimensional nonlinear system of difference equations. Discrete Dyn. Nat. Soc. 2016, Article ID 6842521 (2016)

8. Kocic, V.L., Ladas, G.: Global Behavior of Nonlinear Difference Equations of Higher Order with Applications. Chapman \& Hall, London (1993)

9. Kulenovic, M.R.S., Ladas, G.: Dynamics of Second-Order Rational Difference Equations with Open Problems and Conjecture. Chapman \& Hall, London (2002)

10. Kulenovic, M.R.S., Nurkanovic, M.: Asymptotic behavior of a competitive system of linear fractional difference equations. Adv. Differ. Equ. 2006, Article ID 19756 (2006)

11. Öztürk, Ö:: On the existence of nonoscillatory solutions of three-dimensional time scale systems. J. Fixed Point Theory Appl. 19, 2617-2628 (2017)

12. Öztürk, Ö:: Classification schemes of nonoscillatory solutions for two-dimensional time scale systems. Math. Inequal. Appl. 20(2), 377-387 (2017)

13. Öztürk, Ö.: On oscillatory behavior of two-dimensional time scale systems. Adv. Differ. Equ. 2018, 18 (2018)

14. Papaschinopoulos, G., Schinas, C.J.: On a system of two difference equations. J. Math. Anal. Appl. 219(2), 415-426 (1998)

15. Papaschinopoulos, G., Schinas, C.J.: On the system of two nonlinear difference equations $x_{n+1}=A+\frac{x_{n-1}}{y_{n}}$, $y_{n+1}=A+\frac{y_{n-1}}{x_{n}}$. Int. J. Math. Math. Sci. 23(12), 839-848 (2000)

16. Taşdemir, E., Soykan, Y.: On the periodicies of the difference equation $x_{n+1}=x_{n} x_{n-1}+\alpha$. Karaelmas Sci. Eng. J. 6(2), 329-333 (2016)

17. Tollu, D.T., Yazlık, Y., Taşkara, N.: On fourteen solvable systems of difference equations. Appl. Math. Comput. 233 310-319 (2014) 
18. Yazlık, Y., Elsayed, E.M., Taşkara, N.: On the behaviour of the solutions of difference equation systems. J. Comput. Anal. Appl. 16, 932-941 (2014)

19. Zhang, D., Ji, W., Wang, L., Li, X.: On the symmetrical system of rational difference equation $x_{n+1}=A+\frac{y_{n-k}}{y_{n}}$, $y_{n+1}=A+\frac{x_{n-k}}{x_{n}}$. Appl. Math. 4(5), 834-837 (2013)

20. Zhang, Q., Liu, J., Luo, Z:: Dynamical behavior of a system of third-order rational difference equation. Discrete Dyn. Nat. Soc. 2015, Article ID 530453 (2015)

21. Zhang, Y., Yang, X., Evans, D.J., Zhu, C.: On the nonlinear difference equation system $x_{n+1}=A+\frac{y_{n-m}}{x_{n}}, y_{n+1}=A+\frac{x_{n-m}}{y_{n}}$. Comput. Math. Appl. 53(10), 1561-1566 (2007)

Submit your manuscript to a SpringerOpen ${ }^{\circ}$ journal and benefit from:

- Convenient online submission

$\checkmark$ Rigorous peer review

- Open access: articles freely available online

- High visibility within the field

- Retaining the copyright to your article

Submit your next manuscript at $\gg$ springeropen.com 